\title{
Design-ballistic analysis of a mission for active removal of space debris from geostationary orbit
}

\author{
V.G. Petukhov \\ Research Institute of Applied Mechanics and \\ Electrodynamics \\ Moscow Aviation Institute (National Research \\ University) \\ Moscow, Russian Federation
}

\author{
A.A. Vnukov \\ LLC Small Satellites Center \\ Zheleznogorsk, Krasnoyarsk Krai, Russian Federation \\ vnukovalx@ya.ru
}

\begin{abstract}
The paper considers the service spacecraft (SSC) with electric propulsion system (EPS) to approach the objects of space debris (OSD) and its transportation into a disposal orbit. It suggests the SSC operation algorithm and different ways to ensure its implementation. The paper also provides the equations of SSC motion at optimal control, gives design-ballistic analysis alongside with the calculation of the required resources for the SSC operation.
\end{abstract}

Keywords—spacecraft, space debris, geostationary orbit, service spacecraft, optimal control, fuel budget.

\section{INTRODUCTION}

The prevention of near-space pollution with space debris is one of the most relevant tasks in the attempt to ensure safe access of space flights in the long-term perspective. The exhaust blocks of launch vehicles, now-defunct spacecrafts (SC), dropped elements of launch vehicles typical for the majority of space launches become the objects of space debris (OSD). The geostationary orbit (GSO) is one of the near-earth orbits mostly populated with SC and OSD.

To prevent the GSO pollution, the international rules and national standards imply some measures, including the deorbiting of launch vehicles and worked-out SC from the GSO vicinity. Unfortunately, due to technical reasons, the deorbiting of SC and launch vehicles is not always possible. During the last 50 years of GSO utilization, a large number of OSD has accumulated and their deorbiting is only possible through specially developed technical means, for example the service spacecrafts (SSC), which approach the OSD, ensure its capture and transportation.

According to the Interagency Agreement on the Prevention of Space Pollution, the protected GSO area is the area limited by altitude corresponding to $\pm 200 \mathrm{~km}$ from GSO altitude and by width corresponding to $\pm 15^{\circ}[1,2]$. Taking into account the long-term evolution of an orbit under the influence of natural perturbation, the near-circular orbit located $300 \mathrm{~km}$ higher than the GSO is usually considered a safe disposal orbit.

The paper considers the SSC equipped with electric propulsion system (EPS) to approach the OSD and ensure its transportation to the disposal orbit. It gives preliminary design-ballistic analysis to assess the SSC with regard to EPS time and fuel consumption and hence to the ability to perform major dynamic operations and opportunities of consecutive transportation of a number of OSD from the GSO vicinity into a disposal orbit.

\section{SSC FUNCTIONAL SCHEME AND SOURCE DATA}

The following SSC functional scheme is considered:

1. SSC is launched into a near-circular parking orbit, which altitude is $300 \mathrm{~km}$ higher than the GSO altitude, and the inclination and longitude of ascending node are close to inclination and longitude of the first OSD ascending node, which is to be taken to the disposal orbit. Measuring tools of the SSC ground complex software (GCS) are used at this stage for attitude sensing of the SSC in the orbit.

2. After some time necessary to ensure the SSC phasing with OSD, the SSC flies towards the OSD using the EPS. Besides, the EPS is used to approach the SSC with the OSD up to several kilometers, thus defining the mutual position of the SSC and the OSD by the integrated data of the SSC GCS and the near-space tracking system (for example, automated system to prevent hazardous situations in the near-space).

3. SSC monopropellant hydrazine engines are used on a close maneuvering site and during the OSD capture with the SSC specialized means, and the coordinated referencing is ensured through the range equipment, which each SSC shall be equipped with (a radio range finder or a lidar).

4. After the OSD is captured, the SSC transfers from the OSD into the near-circular disposal orbit, which altitude is 300 $\mathrm{km}$ higher the GSO. The EPS ensures transfer to the orbit, while the SSC OSD controls the SSC GCS trajectory.

5. In the disposal orbit the OSD detaches from the SSC, which, in turn, via the monopropellant hydrazine engines is taken to safe distance several kilometers away from the OSD thus excluding their collision. The distance between the SSC and the OSD is controlled by the SSC GCS.

6. SSC repeats operations starting from item 2 with the next OSD until the depletion of fuel or onboard equipment of the SSC.

For design estimates it is assumed that each OSD represents a worked-out SC not transferred by its propulsion system into the disposal orbit. The mass of each OSD is taken to be equal to $2,000 \mathrm{~kg}$. 
The original SSC mass is taken to be equal to $1,000 \mathrm{~kg}$. For far rendezvous of the SSC with the OSD, the EPS SC consisting of one SPD-100 electric propulsion engine is used. The EPS thrust is taken to be equal to $83 \mathrm{mN}$, the specific impulse - 1,520 s.

The approach and capture of each OSD are ensured via monopropellant hydrazine engines, which specific impulse is taken to be equal to $220 \mathrm{~s}$ and the required reserves of delta- $\mathrm{V}$ are evaluated at $10 \mathrm{~m} / \mathrm{s}$.

\section{EVOLUTION OF OSD ORBITS IN THE GSO VICINITY AND ASSESSMENT OF OSD ORBITAL PARAMETERS}

The analysis of long-term evolution of the OSD in the GSO vicinity under the influence of major perturbing factors (gravitational attraction of moon and sun, non-centrality of the earth's gravitational field, light pressure) states that when the OSD is formed on the GSO its orbit remains near-circular and the period is close to a star day [3]. The major semi-axis of the OSD orbit deviates to not more than $\pm 20 \mathrm{~km}$ from the GSO altitude with the period slightly exceeding 2 years. Longperiod perturbations lead to eccentricity change in the range from $0.00002 \ldots 0.00035$ to $0.0015 \ldots 0.0023$ over the oneyear period, and the amplitude of these oscillations varies throughout the period of about 10 years. The mean eccentricity of the OSD orbit at a large time interval may be taken to be equal to 0.001 .

Throughout the precession period of the near-geostationary orbit ( 53.4 years) the inclination of the orbit plane towards the equator plane is first increases approximately to $15^{\circ}$ and then decreases to near-zero values. The longitude of ascending node for the same period steadily decreases from plus $90^{\circ}$ to minus $90^{\circ}$. Figure 1 shows the inclination vector $\operatorname{hodograph}\left(i_{x}, i_{y}\right)=\left(\tan \frac{i}{2} \cos \Omega, \tan \frac{i}{2} \sin \Omega\right)$, (where $i-$ inclination, and $\Omega$ - longitude of ascending OSD orbit node) in the interval of 53.4 years at passive movement of the OSD located on the GSO at the initial time.

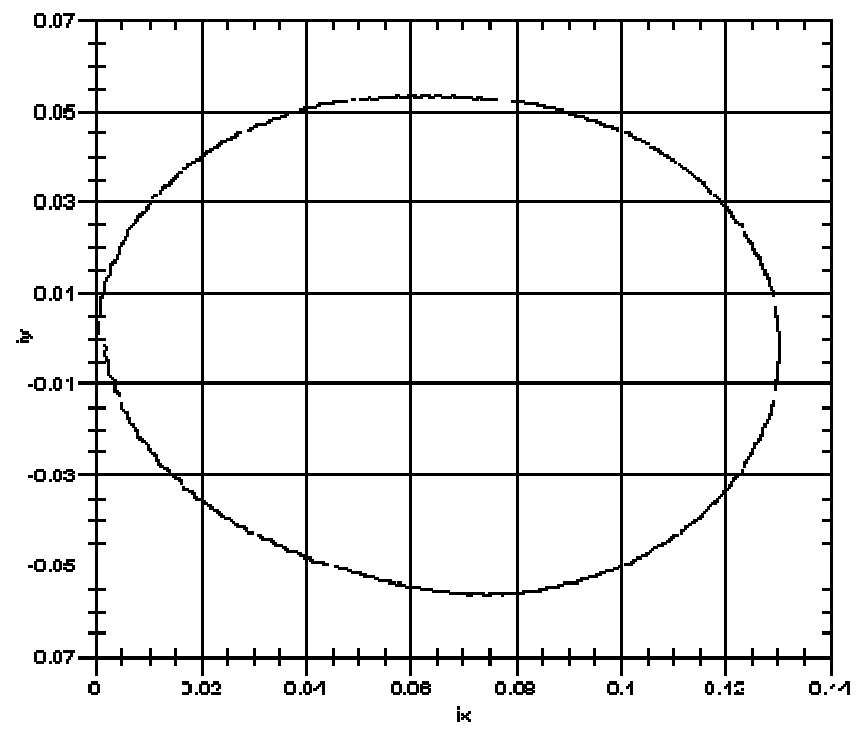

Fig. 1. Inclination vector hodograph in the interval of 53.4 years at passive movement of the OSD located on the GSO at the initial time
The inclination vector hodographs of various OSD are close, and the current arrangement of the OSD on the hodograph is defined by time interval from the moment of a stand point width breakdown of a SC, which turned into the given OSD. Then, the majority of OSD representing geostationary SC that stopped functioning are located along the hodograph shown in Figure 1, and the angle between the orbit planes of the neighboring OSD does not exceed $1-2^{\circ}$.

Thus, let us consider the OSD orbits with major semi-axis $42164 \pm 20 \mathrm{~km}$, maximum eccentricity 0.0023 , mean eccentricity 0.001 , inclination in the range from 0 to $15^{\circ}$ and longitude of ascending node from minus 90 to plus $90^{\circ}$. At the same time it should be assumed that the angle between the orbit planes of consistently transported OSD does not exceed $2^{\circ}$.

\section{CALCULATION OF FLIGHT TRAJECTORIES OF SERVICE SC FROM THE WAITING ORBIT TO THE OSD}

As a rule, the trajectories of interorbital flights of the EPSbased SC are characterized by a big range angle. To increase the calculation velocity and accuracy of numerical integration of such trajectories it is suggested to use differential equations of the SC disturbance motion written in osculating orbital elements. From a variety of options regarding the set of orbital elements it is advisable to use equinoctial orbital elements having no features in the vicinity of circular and equatorial orbits, in particular, in the GSO vicinity.

The undisturbed motion equations of EPS-based SC in equinoctial elements in inertial earth centered equatorial system J2000 are as follows [4]:

$\frac{d h}{d t}=\delta \frac{T}{m} \frac{h}{\xi} \cdot h \cos \vartheta \cos \psi$

$\frac{d e_{x}}{d t}=\delta \frac{T}{m} \frac{h}{\xi}\left\{\xi \sin F \sin \vartheta \cos \psi+\left[(\xi+1) \cos F+e_{x}\right] \cos \vartheta \cos \psi-e_{y} \eta \sin \psi\right\}$,

$\frac{d e_{y}}{d t}=\delta \frac{T}{m} \frac{h}{\xi}\left\{-\xi \cos F \sin \vartheta \cos \psi+\left[(\xi+1) \sin F+e_{y}\right] \cos \vartheta \cos \psi+e_{x} \eta \sin \psi\right\}$,

$\frac{d i_{x}}{d t}=\delta \frac{T}{m} \frac{h}{\xi} \cdot \frac{1}{2} \tilde{\varphi} \cos F \sin \psi$,

$\frac{d i_{y}}{d t}=\delta \frac{T}{m} \frac{h}{\xi} \cdot \frac{1}{2} \tilde{\varphi} \sin F \sin \psi$,

$\frac{d F}{d t}=\frac{\xi^{2}}{h^{3}}+\delta \frac{T}{m} \frac{h}{\xi} \cdot \eta \sin \psi$

$\frac{d m}{d t}=-\delta \frac{T}{c}$,

where $\vartheta$ - pitch angle (angle between a thrust vector projection to the plane of the SC osculating orbit and transversal direction), $\psi$ - yaw angle (angle between a thrust vector and a plane of the SC osculating orbit), $h=\sqrt{\frac{p}{\mu}}$, $e_{x}=e \cos (\Omega+\omega), \quad e_{y}=e \sin (\Omega+\omega), \quad i_{x}=\tan \frac{i}{2} \cos \Omega$, $i_{y}=\tan \frac{i}{2} \sin \Omega$ and $F=v+\omega+\Omega-$ equinoctial elements, $p$ - focal parameter, $e$ - eccentricity, $\omega$ - argument of perigee, $i$ - inclination, $\Omega$ - longitude of ascending node, $v$ - true anomaly, $\xi=1+e_{x} \cos F+e_{y} \sin F, \eta=i_{x} \sin F-i_{y} \cos F$, $\widetilde{\varphi}=1+i_{x}^{2}+i_{y}^{2}$. 
Projections of the EPS jet acceleration to unit vectors of orbital coordinate system are as follows:

$a_{\tau}=\delta \frac{P}{m} \cos \vartheta \cos \psi, a_{r}=\delta \frac{P}{m} \sin \vartheta \cos \psi, a_{n}=\delta \frac{P}{m} \sin \psi$

where $a_{\tau}, a_{r}, a_{n}$ - respectively transversal, radial and binormal projections of jet acceleration.

There is a need to transfer SC of the initial mass $m_{0}$ from the initial orbit

$$
h=h_{0}, e_{x}=e_{x 0}, e_{y}=e_{y 0}, i_{x}=i_{x 0}, i_{y}=i_{y 0}
$$

to the target orbit

$$
h=h_{k}, e_{x}=e_{x k}, e_{y}=e_{y k}, i_{x}=i_{x k}, i_{y}=i_{y k}
$$

in a minimum time $t_{f}$.

The problem of minimizing the functional is thus considered

$$
J=\int_{0}^{t_{f}} d t \rightarrow \min
$$

The maximum principle is used to solve the problem (1), (3)-(5).

The Hamiltonian of the considered optimal control problem is as follows [4]

$H=-1+\frac{\xi^{2}}{h^{3}} p_{F}+\delta \frac{T}{m} \frac{h}{\xi}\left(A_{\tau} \cos \vartheta \cos \psi+A_{r} \sin \vartheta \cos \psi+A_{n} \sin \psi\right)-\delta \frac{T}{c} p_{m}$

where

$$
\begin{aligned}
A_{\tau}=h p_{h}+\left[(\xi+1) \cos F+e_{x}\right] p_{e x}+\left\lfloor(\xi+1) \sin F+e_{y}\right] p_{e y}, \\
A_{r}=\xi\left(\sin F \cdot p_{e x}-\cos F \cdot p_{e y}\right), \\
\quad A_{n}=\eta\left(-e_{y} p_{e x}+e_{x} p_{e y}\right)+\frac{1}{2} \tilde{\varphi}\left(\cos F \cdot p_{i x}+\sin F \cdot p_{i y}\right)+\eta \cdot p_{F},
\end{aligned}
$$

and $p_{h}, p_{e x}, p_{e y}, p_{i x}, p_{i y}, p_{F}, p_{m}$ - variables conjugate to phase coordinates, $h, e_{x}, e_{y}, i_{x}, i_{y}, F$ and $m$ respectively.

Optimal control $\delta(t), \vartheta(t), \psi(t)$ is defined according to the condition of the Hamiltonian maximum (6):

$$
\begin{aligned}
& \cos \vartheta=\frac{A_{\tau}}{\sqrt{A_{\tau}^{2}+A_{r}^{2}}}, \quad \sin \vartheta=\frac{A_{r}}{\sqrt{A_{\tau}^{2}+A_{r}^{2}}} \\
& \cos \psi=\frac{\sqrt{A_{\tau}^{2}+A_{r}^{2}}}{\sqrt{A_{\tau}^{2}+A_{r}^{2}+A_{n}^{2}}}, \sin \psi=\frac{A_{n}^{2}}{\sqrt{A_{\tau}^{2}+A_{r}^{2}+A_{n}^{2}}}, \\
& \delta=\left\{\begin{array}{l}
1, \psi_{s}>0 \\
0, \psi_{s} \leq 0,
\end{array}\right.
\end{aligned}
$$

where $\psi_{s}=-\frac{p_{m}}{c}+\frac{h}{m \xi}\left(A_{\tau}^{2}+A_{r}^{2}+A_{n}^{2}\right)^{1 / 2}-$ switching function. In the problem on flight within a minimum time, taking into account the transversability condition $p_{m}\left(t_{f}\right)=0$ and nonnegativity constraint $d p_{m} / d t$, the condition (9) results in equality

$\delta \equiv 1$,

thus, the differential equations for variables $m$ and $p_{m}$ may be neglected using explicit dependence of the SC mass $m$ on time:

$$
m=m_{0}-(T / c) \cdot t
$$

The substitution of expressions for optimal control (7), (8) and (10) in (6) taking into account (11) leads to the expression for the optimal Hamiltonian:

$$
\begin{aligned}
& H=-1+\frac{T}{m(t)} \frac{h}{\xi}\left(A_{\tau}^{2}+A_{r}^{2}+A_{n}^{2}\right)+\frac{\xi^{2}}{h^{3}} p_{F}=k T A+H_{F}, \quad(12) \\
& \text { where } \quad k=\frac{1}{m(t)} \frac{h}{\xi}, \quad A=\left(A_{\tau}^{2}+A_{r}^{2}+A_{n}^{2}\right)^{1 / 2},
\end{aligned}
$$

$H_{F}=\frac{\xi^{2}}{h^{3}} p_{F}$

Thus, the optimal motion equations will be as follows:

$$
\begin{aligned}
\frac{d \mathbf{x}}{d t} & =\frac{\partial H}{\partial \mathbf{p}}=\delta T\left[k\left(A_{\tau} \frac{\partial A_{\tau}}{\partial \mathbf{p}}+A_{r} \frac{\partial A_{r}}{\partial \mathbf{p}}+A_{n} \frac{\partial A_{n}}{\partial \mathbf{p}}\right) A^{-1}\right], \\
\frac{d F}{d t} & =\frac{\partial H}{\partial p_{F}}=\delta T\left[k\left(A_{\tau} \frac{\partial A_{\tau}}{\partial p_{F}}+A_{r} \frac{\partial A_{r}}{\partial p_{F}}+A_{n} \frac{\partial A_{n}}{\partial p_{F}}\right) A^{-1}\right]+\frac{\partial H_{F}}{\partial p_{F}}, \\
\frac{d \mathbf{p}}{d t} & =-\frac{\partial H}{\partial \mathbf{x}}=-\delta T\left[\frac{\partial k}{\partial \mathbf{x}} A+k\left(A_{\tau} \frac{\partial A_{\tau}}{\partial \mathbf{x}}+A_{r} \frac{\partial A_{r}}{\partial \mathbf{x}}+A_{n} \frac{\partial A_{n}}{\partial \mathbf{x}}\right) A^{-1}\right]-\frac{\partial H_{F}}{\partial \mathbf{x}}, \\
\frac{d p_{F}}{d t} & \left.=-\frac{\partial H}{\partial F}=-\delta T\left[\frac{\partial k}{\partial F} A+k\left(A_{\tau} \frac{\partial A_{\tau}}{\partial F}+A_{r} \frac{\partial A_{r}}{\partial F}+A_{n} \frac{\partial A_{n}}{\partial F}\right) A^{-1}\right]-\frac{\partial H_{F}}{\partial F} \cdot\right) \\
& \text { where } \mathbf{x}=\left(h, e_{x}, e_{y}, i_{x}, i_{y}\right)^{\mathrm{T}}, \mathbf{p}=\left(p_{h}, p_{e x}, p_{e y}, p_{i x}, p_{i y}\right)^{\mathrm{T}} .
\end{aligned}
$$

Since the interorbital flight with a free range angle is considered, the true longitude $F$ in a target orbit is not fixed and hence $p_{F}\left(t_{f}\right)=0$. The optimal Hamiltonian (12) after true longitude averaging does not depend on $F$ and hence $\frac{d p_{F}}{d t}=-\frac{\partial H}{\partial F}=0$. Therefore, according to the averaging solution $p_{F} \equiv 0$. Considering the apparent averaging, the optimal Hamiltonian (12) is as follows:

$$
H=-1+k T A,
$$

and the motion equations (13) may be rewritten as follows:

$$
\left.\begin{array}{l}
\frac{d \mathbf{x}}{d t}=\frac{\partial H}{\partial \mathbf{p}}=T\left[k\left(A_{\tau} \frac{\partial A_{\tau}}{\partial \mathbf{p}}+A_{r} \frac{\partial A_{r}}{\partial \mathbf{p}}+A_{n} \frac{\partial A_{n}}{\partial \mathbf{p}}\right) A^{-1}\right], \\
\frac{d \mathbf{p}}{d t}=-\frac{\partial H}{\partial \mathbf{x}}=-T\left[\frac{\partial k}{\partial \mathbf{x}} A+k\left(A_{\tau} \frac{\partial A_{\tau}}{\partial \mathbf{x}}+A_{r} \frac{\partial A_{r}}{\partial \mathbf{x}}+A_{n} \frac{\partial A_{n}}{\partial \mathbf{x}}\right) A^{-1}\right] \cdot
\end{array}\right\}
$$

Due to smallness of the relation of jet acceleration to gravitational it is possible to use the averaging of differential optimal motion equations. The averaging allows considerably increasing the step length of numerical integration and thus reducing the computing complexity of the problem. However, the main aspect of applying averaging in the given problem is its regularized role: the averaged system of differential optimal motion equations is numerically more stable than the nonaveraged one.

Time-averaging within the orbital period of the SC is used, which corresponds to averaging by mean anomaly of the SC, known in gravitational astronomy [5]. The asymptotic justification of this scheme is well understood: the solution of differential equations averaged following this scheme is a zero term of expansion of the solution of non-averaged equations into the Fourier series according to multiple of the SC mean 
anomaly. The intuitive justification of this averaging scheme covers insignificant changes of slow elements of the SC orbit influenced by jet acceleration per one round.

Averaging of differential equations is made according to the formula:

$$
\frac{d \mathbf{y}}{d t}=\frac{1}{T_{p}} \int_{t_{0}}^{t_{0}+T_{p}} \mathbf{f}_{e}(\mathbf{y}, F, t) d t=\frac{n}{2 \pi} \int_{0}^{2 \pi} \mathbf{f}_{e}(\mathbf{y}, F, t) \frac{d t}{d F} d F,
$$

where $T_{p}$ - osculating period, $\mathbf{y}=\left(\mathbf{x}^{\mathrm{T}}, \mathbf{p}^{\mathrm{T}}\right)^{\mathrm{T}}$ for the problem of optimal fast action, $\mathbf{y}=\left(\mathbf{x}^{\mathrm{T}}, m, \mathbf{p}^{\mathrm{T}}, p_{m}\right)^{\mathrm{T}}$ for a fixed-time problem, $\mathbf{f}_{e}(\mathbf{y}, F, t)$ - right sides of non-averaged differential equations (15), $\quad n=\frac{1}{\mu}\left[\sqrt{1-e_{x}^{2}-e_{y}^{2}} / h\right]^{\beta}-$ mean motion, $d t / d F=h^{3} / \xi^{2}$.

As a result of integration of equations (15) using averaging (16) the phase vector values (in this case - a vector of osculating equinoctial elements and mass of the SC) $\mathbf{x}$ and a vector of conjugate variables $\mathbf{p}$ in final time $t_{f}$, and values of residuals of the boundary value problem are defined:

$$
\mathbf{f}=\left(\begin{array}{l}
h\left(t_{f}\right)-h_{k} \\
e_{x}\left(t_{f}\right)-e_{x k} \\
e_{y}\left(t_{f}\right)-e_{y k} \\
i_{x}\left(t_{f}\right)-i_{x k} \\
i_{y}\left(t_{f}\right)-i_{y k} \\
H\left(t_{f}\right)
\end{array}\right)=0 .
$$

The equations (17) shall be solved with regard to the vector of unknown parameters of a boundary value problem, which for the problem of optimal fast action is as follows:

$$
\mathbf{z}=\left(\begin{array}{l}
\mathbf{p} \\
t_{f}
\end{array}\right) .
$$

The flight between circular orbits presents a particular interest with regard to the considered problem on the OSD deorbiting from the GSO vicinity. If the initial and target orbits are coplanar, the optimal direction of a thrust vector in the averaged problem matches the transversal direction (pitch and yaw angles equal zero along the entire flight trajectory), and the delta- $\mathrm{V}$ of a flight is equal to the difference of orbital velocity in initial and target orbits. If the orbits are noncoplanar, but the angle between planes of initial and target orbits $\Delta i$ is small, then the optimal pitch angle is equal to zero, and the yaw angle represents the periodic function of true longitude. If $\Delta i<5^{\circ}$ and within the considered range of radiuses of initial and target orbits $( \pm 1000 \mathrm{~km}$ from the GSO radius), the oscillation amplitude along the yaw angle almost does not change over a flight.

The averaged equations give a big error when calculating a fast variable - a true longitude of the SC at the end of a flight. Therefore, to calculate the range angle of a flight the nonaveraged motion equations are integrated in the form of (13). The initial values for conjugate variables $\mathbf{p}$ represent their averaged values obtained through the boundary optimal control problem for averaged motion equations, and the initial value for $p_{F}$ is taken to be equal to 0 .

\section{ASSESSMENT OF POSSIBILITY TO REMOVE SEVERAL OSD FROM THE GSO VICINITY TO A DISPOSAL ORBIT}

Let us consider the possibility of consecutive deorbiting of several OSD using one SSC from the GSO vicinity into a near-circular disposal orbit, which is $300 \mathrm{~km}$ higher than the GSO orbit. It is assumed that the SSC flight from a disposal orbit (also referred to as the waiting orbit) to the OSD is done in three stages. At the first stage the SSC using the EPS flies from the waiting orbit into a circular orbit with major semiaxis, inclination and longitude of ascending node coinciding with the corresponding orbital parameters of the OSD. To bring the SSC to the OSD vicinity there is a need to ensure matching of mean longitudes of the SSC and the OSD at the end of the first stage of a flight. The fulfillment of this condition is guaranteed by the SSC phasing in the waiting orbit during the required time interval (mean drift velocity of the SSC in relation to the OSD located on an orbit over a diurnal period is $3.819^{\circ}$ per day). The boundary optimal control problem described above is solved to define the flight parameters. At the second stage the SSC using the EPS levels the eccentricity and longitude of a perigee. For this the thrust vector of the SSC EPS is oriented in the orbit plane orthogonally to the apse line of the OSD orbit. The direction of the EPS thrust vector remains invariable in inertial space and the EPS operates in apogee and perigee sites with equal range angle of eccentric anomaly. At the end of the second stage the SSC approaches the OSD at a distance of several kilometers. Further approach of the SSC with the OSD until its capture (third stage) is carried out by the monopropellant hydrazine propulsion system. The required delta- $\mathrm{V}$ for the third stage is estimated at $10 \mathrm{~m} / \mathrm{s}$, and the stage duration -10 days. Upon capturing the OSD the SSC transfers it to the disposal orbit being in the plane of the initial OSD orbit (fourth stage). The transfer is ensured by continuously operating SSC EPS, the EPS thrust is in transversal direction.

Table 1 shows the main characteristics of the first stage of the SSC flight from the waiting orbit to the first OSD depending on the OSD orbit plane inclination to the plane of the SSC waiting orbit (initial SSC mass is equal to $1,000 \mathrm{~kg}$ ). The initial phase angle in this table is understood as the difference between mean longitudes of the OSD and the SSC at the beginning of a flight thus ensuring their equality in the end of a flight. The EPS engine time is equal to the minimum duration of the first stage of a flight (is applicable in case of equality of the initial phase angle to the required value). The maximum time of a flight includes phasing time at $360^{\circ}$ and flight time from the operating EPS (engine time). 
TABLE I. MAIN CHARACTERISTICS OF THE FIRST STAGE OF THE SSC TRANSFER FROM THE WAITING ORBIT TO THE FIRST OSD DEPENDING ON THE OSD ORBIT PLANE INCLINATION TO THE PLANE OF THE SSC WAITING ORBIT

\begin{tabular}{|c|c|c|c|c|}
\hline 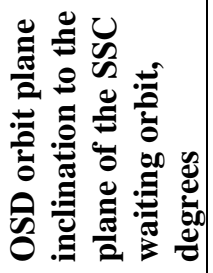 & 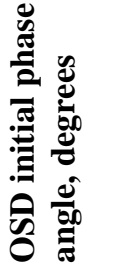 & 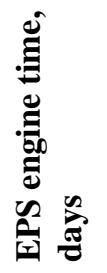 & 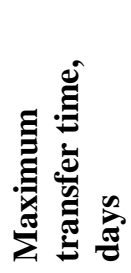 & 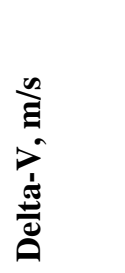 \\
\hline 0 & -2.795 & 1.517 & 95.791 & 10.879 \\
\hline 1 & -21.653 & 11.750 & 106.024 & 84.499 \\
\hline 2 & -43.032 & 23.351 & 117.625 & 168.399 \\
\hline
\end{tabular}

The delta- $\mathrm{V}$ of the second stage makes $4.714 \mathrm{~m} / \mathrm{s}$ at the maximum eccentricity of the OSD orbit (0.0023) and 2.050 $\mathrm{m} / \mathrm{s}$ at mean eccentricity $(0.001)$. The second stage does not exceed 2 days.

The delta- $\mathrm{V}$ of the third stage, as it was stated above, is taken to be equal to $10 \mathrm{~m} / \mathrm{s}$, and duration -10 days.

The delta- $\mathrm{V}$ of the fourth stage is $10.880 \mathrm{~m} / \mathrm{s}$, duration less than 4.6 days.

Table 2 shows the required consumption of xenon, hydrazine and time for the first cycle of the SSC (flight from the waiting orbit to the OSD and its transportation into the disposal orbit) depending on the OSD orbit plane inclination to the plane of the SSC waiting orbit. The OSD orbit eccentricity is taken to be equal to the mean value (0.001).

TABLE II. REQUIRED CONSUMPTION OF XENON, HYDRAZINE AND TIME FOR THE FIRST CYCLE OF THE SSC

\begin{tabular}{|c|c|c|c|}
\hline 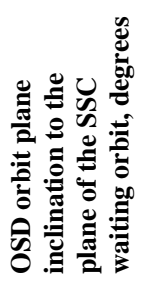 & 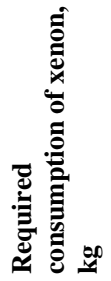 & 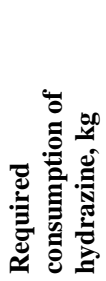 & 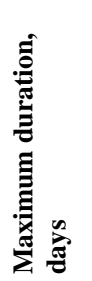 \\
\hline 0 & 3.052 & 4.620 & 112.4 \\
\hline 1 & 7.971 & 4.598 & 122.6 \\
\hline 2 & 13.547 & 4.572 & 134.2 \\
\hline
\end{tabular}

At each new cycle the initial SSC mass decreases due to the consumption of xenon and hydrazine thus decreasing the required consumption of working fuel and time in each subsequent cycle of the SSC. Less than 7.35 years of the SSC functioning is required to remove 20 OSD. In this case the required xenon mass changes from 61.04 to $270.94 \mathrm{~kg}$, and hydrazine - from 92.4 to $104.4 \mathrm{~kg}$, depending on the orientation of the OSD orbital planes and their service arrangement. The minimum fuelling of a propellant is ensured by the coincidence of orbital planes of the SSC and the OSD, and the maximum - by the $2^{\circ}$ difference of inclinations of the SSC orbits and each subsequent OSD.

\section{CONCLUSIONS}

The preliminary design-ballistic analysis of the SSC functioning regarding active removal of space debris from the geostationary orbit suggests the SSC scheme implying five main stages, each of which containing the SSC and the OSD trajectories control systems, as well as the type of the propulsion system used for maneuvering.

The analysis of long-term evolution of quasi-geostationary orbits made it possible to define the range of the OSD orbits applicable for the SSC. The major semi-axis of these orbits falls within $42164 \pm 20 \mathrm{~km}$, the maximum eccentricity is 0.0023 , the mean eccentricity -0.001 , and the inclination shall be in the range from $0^{\circ}$ to $15^{\circ}$. The calculations of propellant costs required to transfer the OSD into the disposal orbit were made assuming that the angle between the orbit planes of consistently transported OSD does not exceed $2^{\circ}$.

The maximum principle was used to obtain the system of optimal control equations allowing minimizing the propellant costs for the SSC maneuvering both at its independent movement and at joint movement with the OSD following the undisturbed motion equations of EPS-based SC in equinoctial elements in inertial earth centered equatorial system of coordinates.

The preliminary design-ballistic analysis of the SSC motion with optimal control demonstrates the possibility of transferring at least 20 OSD $(2,000 \mathrm{~kg}$ each) from the GSO vicinity into disposal orbits thus the lifetime of the SSC shall be not less than 7.5 years. The longer lifetime will provide for service interruption of the SSC operation, which can be used to manage emergency situations during orbital operation.

\section{Acknowledgment}

The study is carried out within the applied research on the Design of Service Spacecraft for Treatment of Geostationary Orbit (GSO) Area from Technogenic Space Debris under financial support of the Ministry of Education and Science of the Russian Federation, Agreement No. RFMEFI57617X0093.

\section{References}

[1] IADC Space Debris Mitigation Guidelines, Inter-agency Space Debris Coordination Committee site. IADC-02-01, 2007. Retrieved from: http://www.iadc-online.org/Documents/IADC-200201,\%20IADC\%Space\%20Debris\%20Guidelines,\%20Revision\%201.pdf

[2] GOST P 52925-2008. Products of space engineering. General requirements to space systems on the restriction of technogenical contamination of the near-space. Intr. 2009-01-01. M., pp. 7.

[3] A.G. Alexandrova, Study of long-term orbital evolution of space debris of geostationary zone: Cand. Sci. (Math.) Dissertation: 01.03.01 / St. Petersburg State University, St. Petersburg, 2012.

[4] V.G. Petukhov "Optimization of multi-orbit transfers between noncoplanar elliptic orbits”, Cosmic Research, Vol. 42 (3) , pp. 250-268, 2004

[5] G.N. Duboshin, Reference manual on gravitational astronomy and astrodynamics, M.: NauSC. Fizmatlit Publishing House, 1976. 